\title{
Evolución de la lumbalgia en el paciente oncológico tratado con manejo intervencionista del dolor
}

\author{
Evolution of low back pain in cancer patients treated with \\ interventional pain management
}

X. E. Sacoto García, R. Guillén Núñez y A. Juárez Lemus

Clínica de Dolor. Instituto Nacional de Cancerología, Ciudad de México, México

\section{RESUMEN}

Objetivo: Identificar la evolución de la lumbalgia en el paciente oncológico tratado con manejo intervencionista del dolor.

Material y métodos: Estudio descriptivo y retrospectivo. Se realizó búsqueda en la base de datos de los pacientes atendidos en la Clínica del dolor del Instituto Nacional de Cancerología sometidos a procedimiento intervencionista por lumbalgia en el periodo de enero de 2017 a diciembre de 2019. El análisis estadístico se realizó en el programa SPSS 15.0 y la información se presentó en tablas de frecuencia.

Resultados: Se analizaron 143 pacientes quienes fueron en mayor porcentaje mujeres (69 \%) con una media de edad de 65 años. Los factores de riesgo identificados más frecuentes fueron diabetes mellitus tipo 2 (18,2\%) e hipertensión arterial (14,7\%). Los diagnósticos oncológicos de mayor frecuencia fueron cáncer de mama $(38,5 \%)$, cáncer de próstata (14\%), mieloma múltiple $(13,3 \%$ y cáncer cervicouterino $(10,5 \%)$. Los opioides utilizados fueron tramadol $(48,3 \%)$, morfina [32,2 \%), buprenorfina y tapentadol $(5,6 \%)$, oxicodona $[1,4 \%)$, fentanilo y metadona $[0,7 \%)$. Los abordajes intervencionistas en mayor proporción fueron depósito de esteroides en ramo dorso medial de articulación cigoapofisiaria lumbar de 3 segmentos $(11,2 \%$ ), depósito de esteroides de ramo dorso medial de articulación cigoapofisiaria lumbar de 4 o más segmentos el $[7,7 \%)$ y vertebroplastia $[5,6 \%)$. Además se observó que el $60,8 \%$ de los pacientes recibió más de un abordaje intervencionista. La respuesta terapéutica mayor o igual al $50 \%$ se presentó en el 86,7\% de los pacientes y el 35,7 \% mostró una media de reducción de opioides
Sacoto García XE, Guillén Núñez R, Juárez Lemus A. Evolución de la lumbalgia en el paciente oncológico tratado con manejo intervencionista del dolor. Rev Soc Esp Dolor. 2021;28(2):76-81

\section{ABSTRACT}

Objective: To identify the evolution of low back pain in cancer patients treated with interventional pain management.

Material and methods: Descriptive and retrospective study. A search was carried out in data patients treated at Pain Clinic of the National Cancer Institute who underwent an interventional procedure for low back pain in the period from January 2017 to December 2019. The statistical analysis was performed in SPSS 15.0 program and information was presented in frequency tables.

Results: 143 patients were analyzed, the highest percentage being women (69\%) with a mean age of 65 years. Most frequent risk factors identified were type 2 diabetes mellitus (18.2\%) and arterial hypertension (14.7\%). Most frequent oncological diagnoses were breast cancer (38.5\%), prostate cancer (14\%), multiple myeloma (13.3\%), and cervical cancer (10.5\%). Opioids used were tramadol (48.3\%), morphine (32.2\%), buprenorphine and tapentadol (5.6\%), oxycodone (1.4\%), fentanyl and methadone (0.7\%). Interventional approaches in greater proportion were steroid deposition in dorsal medial branch of the lumbar zygapophyseal joint of 3 segments (11.2\%), steroid deposition of dorsal medial branch of the lumbar zygapophyseal joint of 4 or more segments (7.7\%) and vertebroplasty (5.6\%), it was also observed that $60.8 \%$ of patients received more than one interventional approach. Therapeutic response greater than or equal to $50 \%$ was presented in $86.7 \%$ of patients and $35.7 \%$ showed a mean reduction in opioids of 22.2 milligrams / day. Low back pain with a neuropathic component occurred in $57.3 \%$ of patients. According to verbal analogue scale
Recibido: 08-02-2021

Aceptado: 17-04-2021

Correspondencia: Xavier Esteban Sacoto García xavier_sac@hotmail.com 
de 22,2 miligramos/día. La lumbalgia con componente neuropático se presentó en el $57,3 \%$ de los pacientes. De acuerdo con la evaluación de la escala verbal análoga antes y a la semana postratamiento, se observaron cambios de dolor leve del 8,4 al 77,6\%, dolor moderado del 21,7 al 46,9\% y dolor severo del 44,8 a 0,7\%. Al mes posterior al procedimiento se observó para dolor leve el 70,6\%, dolor moderado 23,8 \% y severo 5,6\%.

En cuanto a la evaluación del cuestionario Douleur Neuropathique-4 ítems antes del abordaje terapéutico, el $42,7 \%$ de los pacientes presentaron una puntuación menor a 4 , mientras que el $57,3 \%$ presentó un puntaje mayor a 4. A la semana el porcentaje fue del 85,3 y $14,7 \%$, respectivamente, y posterior al mes el $84,6 \%$ de los pacientes presentó una puntación menor a 4.

Conclusiones: El depósito de esteroide de ramo dorso medial de articulación cigoapofisiaria lumbar de 3 segmentos, 4 o más segmentos y vertebroplastia fueron los abordajes más frecuentes. La respuesta terapéutica mayor o igual al $50 \%$ se presentó en el $86,7 \%$ de los pacientes, sin embargo debido a la etiología múltiple de este padecimiento administrar diferentes abordajes en un mismo paciente pudo mejorar el dolor lumbar. Esto se vio reflejado en la persistencia del dolor un mes posterior al procedimiento donde se observó un cambio significativo de dolor moderado y severo a dolor leve [70,6\%), así como en la disminución de analgésicos opioides; sin embargo se requieren estudios prospectivos para mayor caracterización de su efectividad. La elección de realizar estas técnicas se recomienda luego de un análisis individualizado de los pacientes para buscar el mayor beneficio y evitar complicaciones.

Palabras clave: Lumbalgia, manejo intervencionista, dolor, cáncer, dolor oncológico. evaluation before and a week after treatment, changes in mild pain were observed from $8.4 \%$ to $77.6 \%$, moderate pain from $21.7 \%$ to $46.9 \%$ and severe pain from $44.8 \%$ to $0.7 \%$. One month after the procedure, $70.6 \%$ were observed for mild pain, $23.8 \%$ moderate pain and $5.6 \%$ severe. Evaluation of Douleur Neuropathique- 4 items questionnaire before therapeutic approach, $42.7 \%$ of patients presented a score lower than 4, while $57.3 \%$ presented a score greater than 4 . After one week percentage was $85.3 \%$ and $14.7 \%$ respectively, and after one month, $84.6 \%$ of patients presented a score lower than 4.

Conclusions: Steroid deposition in dorsal medial branch of the lumbar zygapophyseal joint of 3 segments, 4 or more segments and vertebroplasty were the most frequent approaches. Therapeutic response greater than or equal to $50 \%$ was presented in $86.7 \%$ of patients, however, due to the multiple etiology of this condition, administering different approaches in the same patient could improve lumbar pain, this was reflected in persistence of pain one month after the procedure where a significant change was observed from moderate and severe pain to mild pain (70.6\%) as well as a decrease in opioid analgesics; however, prospective studies are required to further characterize its effectiveness. The choice to perform these techniques is recommended after an individualized analysis of the patients to seek the greatest benefit and avoid complications.

Key words: Low back pain, interventional management, pain, cancer pain.

\section{INTRODUCCIÓN}

La lumbalgia se ha caracterizado como un síntoma, más que una enfermedad, siendo muy común entre la población. Ha sido definida por las guías de manejo del dolor de espalda baja o lumbalgia propuestas por la Cooperación Europea de Ciencia y Tecnología (COST) como una sensación dolorosa o no confortable que se localiza por debajo del margen costal y por encima del límite superior de la región glútea, pudiendo irradiarse hacia uno o ambos miembros pélvicos, donde la mayoría de sus causas son de etiología inespecífica $[90 \%](1,3]$. En 2008 la prevalencia mundial para el dolor lumbar que incluyó 54 países estimó un 18,3 \%, siendo más común en países de altos ingresos, en mujeres que hombres y en aquellas personas entre 40 y 69 años $[4,5]$.

La lumbalgia puede ser un síntoma que acompaña diferentes patologías como estenosis espinal, enfermedad visceral, procesos tumorales per se o actividad metastásica, procesos infecciosos o fracturas por compresión entre otras, por lo que un diagnósti- co diferencial es esencial. La detección de malignidad debe de ser uno de los principales objetivos, ya que las metástasis ocasionadas por varios tipos de cáncer generan tumoraciones a este nivel ocasionando dolor y sugiriendo avance de la enfermedad. En estos casos el diagnóstico diferencial utilizando las banderas rojas en el dolor lumbar es crucial, ya que al menos el $1 \%$ de los pacientes con dolor lumbar presenta cáncer, requiere terapia específica y puede presentar complicaciones como fracturas patológicas, debilidad, pérdida sensorial, paralipsis de intestino o disfunción de vejiga [6-8].

La valoración inicial de esta patología es clínica apoyándose de estudios diagnósticos como la radiografía simple de columna, la cual permite la evaluación de fracturas y anomalías óseas; sin embargo, la resonancia magnética permite identificar de manera específica las fuentes de anomalías nerviosas o de tejidos blandos [8].

El tratamiento de la lumbalgia es de carácter multidisciplinario y multimodal, incluyendo medidas farmacológicas, no farmacológicas, terapia física, terapia ocupacional y abordajes intervencionistas en pacientes 
con puntuaciones elevadas en la intensidad del dolor, limitación funcional severa en procesos agudos o moderada en casos crónicos pese el mejor abordaje terapéutico. Los antinflamatorios no esteroideos (AINE) son el pilar fundamental en la lumbalgia aguda y en procesos crónicos de dolor lumbar se suma el uso de opioides, neuromoduladores, parches de lidocaína, relajantes musculares y estimulación nerviosa. El uso de bloqueos intervencionistas en el dolor lumbar es una alternativa que se utilizó en 1901 por primera vez y contribuyó a la introducción de la guía fluroscópica, facilitando la precisión y seguridad, ya que permite la visualización clara de las estructuras anatómicas, con lo cual la tasa de éxito se incrementa exponencialmente [8].

En los últimos años se ha observado incremento de la incidencia de lumbalgia entre 1,5\% y $36 \%$, (9) sin embargo la heterogeneidad que existe entre los resultados de los estudios epidemiológicos, así como la falta de estudios en pacientes con cáncer, dificulta la agrupación, comparación y descripción de datos. Es por ello que a través de este estudio se identificó la persistencia de lumbalgia en el paciente con cáncer, a quien se le realizó un procedimiento intervencionista.

\section{MATERIALES Y MÉTODOS}

Cuando el proyecto de investigación fue aprobado por el Comité de Ética e Investigación del Instituto Nacional de Cancerología, se buscó en la bitácora de datos de los pacientes atendidos en la Clínica del Dolor y se identificó a todos los pacientes que fueron sometidos a un procedimiento intervencionista por lumbalgia en el periodo de tiempo comprendido de enero de 2017 a diciembre de 2019.

Los criterios de inclusión fueron:

- Pacientes mayores de 18 años y diagnóstico de cáncer, atendidos en la Clínica del Dolor del Instituto Nacional de Cancerología, en México.

- Pacientes con diagnóstico de dolor lumbar.

- Pacientes a quienes se les realizó algún procedimiento intervencionista en el servicio de Clínica del Dolor durante el periodo de tiempo comprendido del 1 de enero de 2017 al 31 de diciembre de 2019.

Los criterios de exclusión fueron:

- Pacientes menores de 18 años.

- Pacientes con diagnóstico diferente a dolor lumbar.

- Pacientes a los cuales no se les realizó procedimiento intervencionista en el servicio de Clínica del DoIor del Instituto Nacional de Cancerología durante el periodo 2017 a 2019.

- Pacientes con historia clínica incompleta.

Una vez obtenido este registro, se analizaron los expedientes de los pacientes sometidos a procedimiento intervencionista por dolor lumbar para la búsqueda de la nota de procedimiento intervencionista de dolor, donde constaba el diagnóstico etiológico del dolor lumbar, si existía componente radicular o no, esquemas terapéuticos y seguimiento, se registró la información obtenida en una base de datos para su posterior análisis. El análisis estadístico se realizó en el Programa SPSS versión 15.0 (StataCorp, College Station, TX, EE. UU.) utilizando estadística descriptiva (media y porcentaje) y la información se presentó en tablas de frecuencia.

\section{RESULTADOS}

Se seleccionaron un total de 179 pacientes, de los cuales se eliminaron 36 por presentar datos incompletos ( $\mathrm{n}: 10$ ), intervenciones en diferentes lugares ( $\mathrm{n}: 20$ ) y por pérdida de seguimiento (n: 6), por lo que se analizaron 143 pacientes. En la Tabla I se presentan los datos demográficos y clínicos de los pacientes donde se observó que el 69 \% fueron del sexo femenino, con una media de edad de 65 años. Los factores de riesgo observados fueron diabetes mellitus tipo 2 en el $18,2 \%$, hipertensión arterial en el 14,7\%, estas dos condiciones en conjunto en el $13,3 \%$, y otros como depresión e hipotiroidismo en el 16,8\%. La evaluación del diagnóstico oncológico mostró cáncer de mama en el 38,5 \%, cáncer de próstata en el $14 \%$, mieloma múltiple en el 13,3\%, cáncer uterino en el 10,5\%, tumores hematopoyéticos en el 8,4 \%, tumores gastrointestinales en el 3,5\%, tumores genitourinarios en el 2,1\%, cáncer de pulmón y tumores de cabeza y cuello en el 1,4\%.

TABLA I

CARACTERÍSTICAS DEMOGRÁFICAS Y CLÍNICAS DE LOS PACIENTES DE ESTUDIO

\begin{tabular}{|l|c|}
\hline \multicolumn{1}{|c|}{$\begin{array}{c}\text { Características } \\
\text { demográficas y clínicas }\end{array}$} & $N{ }^{\circ}[\%]$ \\
\hline Sexo & $44(31)$ \\
\hline Masculino & $99(69)$ \\
\hline Femenino & $65 \pm 29$ \\
\hline Edad \\
\hline Media \\
\hline Factores de riesgo & $26(18,2)$ \\
\hline Diabetes Mellitus 2 & $21(14,7)$ \\
\hline HTA & $19(13,3)$ \\
\hline HTA y DMT2 & $24(16,8)$ \\
\hline Otra & $53(37,1)$ \\
\hline Ninguna \\
\hline Diagnóstico Oncológico Primario \\
\hline CA mama & $55(38,5)$ \\
\hline CA próstata & $20(14)$ \\
\hline Mieloma múltiple & $19(13,3)$ \\
\hline CA uterino & $15(10,5)$ \\
\hline Tumores hematopoyéticos & $12(8,4)$ \\
\hline Tumores gastrointestinales & $5(3,5)$ \\
\hline Tumores genitourinarios & $3(2,1)$ \\
\hline Tumores de cabeza y cuello & $2(1,4)$ \\
\hline CA pulmón & $2(1,4)$ \\
\hline Otros tumores & $10(7)$ \\
\hline
\end{tabular}

HTA: hipertensión arterial. DMT2: diabetes mellitus tipo 2. CA: cáncer 
En la Tabla II se muestran los analgésicos opioides administrados en los pacientes del estudio, los cuales fueron tramadol en el 48,3\% de los pacientes, morfina representa el 32,2 \%, buprenorfina y tapentadol el 5,6\%, oxicodona el 1,4\%, fentanilo y metadona el 0,7\%.

La Tabla III muestra el análisis de los diferentes tipos de abordajes intervencionistas en los pacientes con lumbalgia y cáncer, la cual muestra que el bloqueo foraminal unilateral 1 nivel representó el 0,7\%, bloqueo foraminal unilateral 2 niveles el $1,4 \%$, bloqueo foraminal unilateral 3 o más niveles el 2,1\%, bloqueo foraminal bilateral 2 o más niveles el 1,4\%, depósito de esteroides interlaminar el 1,4\%, depósito de esteroides con abordaje por hiato sacro el 1,4\%, depósito de esteroides en el ramo dorso medial de la articulación cigoapofisiaria lumbar de 3 segmentos el 11,2 \%, depósito de esteroides del ramo dorso medial de la articulación cigoapofisiaria lumbar de 4 o más segmentos el 7,7\%, neurolisis química con abordaje por hiato sacro el $0,7 \%$, radiofrecuencia térmica de ganglio de la raíz dorsal cualquier nivel lumbar unilateral el 0,7 \%, vertebroplastia el $5,6 \%$, bloqueo erector espinal el 2,1\% e infiltración facetaria el 2,8\%. Finalmente se observó que más del $60,8 \%$ de los pacientes recibieron más de un abordaje intervencionista. El dolor asociado a componente neuropático se presentó en el 57,3\% de los pacientes.

En la Tabla IV se muestra la respuesta terapéutica que presentaron los pacientes del estudio posterior a los abordajes intervencionistas, donde se observa que el 86,7\% de los pacientes presentó respuesta mayor o igual al $50 \%$, mientras que el 2,1\% de los no la presentó.

La Tabla V muestra los cambios en la dosificación de opioides posterior al abordaje intervencionista donde se observó que el $64,3 \%$ de los pacientes no presentó modificaciones, mientras que en el 35,7\% hubo una reducción en la dosis total del opioide en 24 horas de 22,2 miligramos de morfina o sus equivalentes.

TABLA ॥

TRATAMIENTO ALGOLÓGICO OPIOIDE Y COMPONENTE NEUROPÁTICO EN LOS PACIENTES DE ESTUDIO

\begin{tabular}{|l|c|}
\hline \multicolumn{1}{|c|}{ Tratamiento algológico } & $N^{\circ}{ }^{\circ}[\%]$ \\
\hline Tramadol & $69(48,3)$ \\
\hline Morfina & $46(32,2)$ \\
\hline Buprenorfina & $8(5,6)$ \\
\hline Tapentadol & $8(5,6)$ \\
\hline Oxicodona & $2(1,4)$ \\
\hline Fentanilo & $1(0,7)$ \\
\hline Metadona & $1(0,7)$ \\
\hline Componente neuropático & $82(57,3)$ \\
\hline Sí & $61(42,7)$ \\
\hline No & $143(100)$ \\
\hline Total
\end{tabular}

TABLA III

\section{TIPO DE ABORDAJE INTERVENCIONISTA REALIZADO A LOS PACIENTES CON DOLOR LUMBAR Y CÁNCER}

\begin{tabular}{|c|c|}
\hline Tipo de abordaje intervencionista & $N .^{\circ}[\%]$ \\
\hline Bloqueo foraminal unilateral 1 nivel & $1(0,7)$ \\
\hline Bloqueo foraminal unilateral 2 niveles & $2(1,4)$ \\
\hline $\begin{array}{l}\text { Bloqueo foraminal unilateral } 3 \text { o más } \\
\text { niveles }\end{array}$ & $3(2,1)$ \\
\hline $\begin{array}{l}\text { Bloqueo foraminal bilateral } 2 \text { o más } \\
\text { niveles }\end{array}$ & $2(1,4)$ \\
\hline Depósito de esteroides interlaminar & $2(1,4)$ \\
\hline $\begin{array}{l}\text { Depósito de esteroides con abordaje } \\
\text { por hiato sacro }\end{array}$ & $2(1,4)$ \\
\hline $\begin{array}{l}\text { Depósito de esteroides en el ramo } \\
\text { dorso medial de la articulación } \\
\text { cigoapofisiaria lumbar de } 3 \\
\text { segmentos }\end{array}$ & $16(11,2)$ \\
\hline $\begin{array}{l}\text { Depósito de esteroides del ramo } \\
\text { dorso medial de la articulación } \\
\text { cigoapofisiaria } 4 \text { o más segmentos }\end{array}$ & $11(7,7)$ \\
\hline $\begin{array}{l}\text { Nerurolisis química con abordaje por } \\
\text { hiato sacro }\end{array}$ & $1(0,7)$ \\
\hline $\begin{array}{l}\text { Radiofrecuencia térmica de ganglio } \\
\text { de la raíz dorsal cualquier nivel } \\
\text { lumbar unilateral }\end{array}$ & $1(0,7)$ \\
\hline Vertebroplastia & $8(5,6)$ \\
\hline Bloqueo erector espinal & $3(2,1)$ \\
\hline Infiltración facetaria & $4(2,8)$ \\
\hline 2 intervenciones o más* & $87(60,8)$ \\
\hline Total & $143(100)$ \\
\hline
\end{tabular}

* Los siguientes abordajes se realizaron en conjunto con algunos de los presentados en la tabla: radiofrecuencia de ganglio dorso medial bilateral, discoplastia, anuloplastia.

TABLA IV RESPUESTA TERAPÉUTICA AL ABORDAJE INTERVENCIONISTA EN LOS PACIENTES CON DOLOR LUMBAR Y CÁNCER

\begin{tabular}{|l|c|}
\hline \multicolumn{1}{|c|}{ Respuesta terapéutica $\%$} & $N^{\circ}{ }^{\circ}$ pacientes $[\%]$ \\
\hline 0 & $3(2,1)$ \\
\hline 10 & $4(2,8)$ \\
\hline 20 & $4(2,8)$ \\
\hline 30 & $4(2,8)$ \\
\hline 40 & $2(1,4)$ \\
\hline$\geq 50$ & $124(86,7)$ \\
\hline Total & $143(100)$ \\
\hline
\end{tabular}


TABLA V

CAMBIOS EN DOSIS DE OPIOIDES POSTERIOR AL ABORDAJE INTERVENCIONISTA EN PACIENTES CON DOLOR LUMBAR Y CÁNCER

\begin{tabular}{|l|c|}
\hline \multicolumn{1}{|c|}{ Cambios en dosis de opioides } & $N^{\circ}$ pacientes $[\%]$ \\
\hline Sin cambios & $92(64,3)$ \\
\hline Con cambios & $51(35,7)$ \\
\hline $\begin{array}{l}\text { Media de reducción de dosis en } \\
\text { miligramos/día de morfina o } \\
\text { equivalentes }\end{array}$ & 22,2 \\
\hline
\end{tabular}

Para la evaluación del dolor en este grupo de pacientes se utilizaron dos escalas, la Tabla VI muestra estos resultados. De acuerdo con la escala verbal análoga (EVERA), el 8,4\% de los pacientes presentaron dolor leve antes del abordaje, el 46,9\% dolor moderado y el $44,8 \%$ dolor severo; a la evaluación de la semana postratamiento intervencionista los porcentajes fueron del 77,6, 21,7 y 0,7\%, respectivamente. La persistencia del dolor un mes posterior al procedimiento en estos pacientes se observó para dolor leve el 70,6\%, dolor moderado el 23,8 \% y severo el 5,6 \%.

Finalmente, de acuerdo con la evaluación del cuestionario Douleur Neuropathique-4 ítems (DN4), antes del abordaje terapéutico el 42,7\% de los pacientes presentaron una puntuación menor a 4 , mientras que el $57,3 \%$ presentó un puntaje mayor a 4 . A la semana el porcentaje fue del $85,3 \%$ y $14,7 \%$ respectivamente, y posterior al mes el 84,6\% de los pacientes presentó una puntación menor a 4.

\section{DISCUSIÓN}

En este estudio se describe la persistencia de lumbalgia en el paciente oncológico con manejo intervencionista del dolor en una población total de 143 pacientes, en quienes pudimos identificar que los factores de riesgo de mayor frecuencia fueron diabetes mellitus tipo 2 e hipertensión arterial. En cuanto los diagnósticos oncológicos más frecuentes observados fueron el cáncer de mama, cáncer de próstata, mieloma múltiple y cáncer de útero; estos resultados concuerdan con lo reportado por Steinberger y cols. (9), ya que se ha reportado que estos tipos de cáncer son los que mayormente presentan metástasis hacia el sistema óseo. Hatrick y cols. también mencionan que las metástasis espinales afectan del 40 al $70 \%$ de los pacientes con cáncer terminal, siendo las regiones lumbar, torácica y cervical las más afectadas $(10,11)$.

Por otro lado, como el objetivo del estudio fue evaluar la persistencia de dolor en este tipo de pacientes, se analizaron los diferentes abordajes intervencionistas realizados obteniendo resultados similares con los reportados por Ruiz Santiago y cols., donde refieren que la articulación facetaria es la responsable del 15-45\% de la etiología del dolor en este segmento de la columna $(12,13) ;$ sin embargo, es importante mencionar que al $60,8 \%$ de los pacientes de nuestro estudio se les realizó más de un procedimiento, debido a que el origen del dolor es etiológicamente multifactorial, y al realizar varios abordajes intervencionistas a estos pacientes se puede obtener una mejoría clínica significativa. Dentro de los procedimientos realizados el abordaje de vertebro plastia, fue de un $5,6 \%$, sin embargo este procedimiento al momento no cuenta con recomendaciones fuertes debido a la baja calidad de los ensayos clínicos aleatorios (ECA) que se tienen al momento (14).

La selección de los pacientes que se deberían realizar un procedimiento intervencionista se basan en el difícil control de estos casos o en el aumento de la ingesta de opioides, los cuales son seleccionados después de una revisión física y un consenso del caso debido a que el dolor no es un proceso unidimensional, por lo que se deben evaluar todos los posibles factores que intervienen en este, es así que Mercante en su artículo (15) manifiesta los peligros que pueden implicar el basarse solamente en la literatura médica sin una correcto enfoque clínico.

Para la evaluación del dolor posterior al procedimiento intervencionista en esta población de estudio se utilizaron dos escalas, la escala EVERA reflejó disminución en el porcentaje de pacientes con dolor moderado y dolor severo, conjuntamente se observó incremento del porcentaje de pacientes que reportaron dolor leve posterior a la intervención. Estos resultados concuerdan con el estudio realizado por Surbano y cols., quienes también evaluaron la reducción del dolor, no obstante la escala utilizada fue Brief Pain Inventory (BPI) a las 24 horas con una mejoría clínica del $50 \%$ [16].

TABLA VI

ESCALAS DE DOLOR EVALUADAS EN LOS PACIENTES DEL ESTUDIO PREVIO AL ABORDAJE INTERVENCIONISTA, A LA SEMANA Y AL MES

\begin{tabular}{|c|c|c|c|c|c|c|c|}
\hline EVERAa & $\begin{array}{l}\text { Antes del } \\
\text { abordaje } \\
N .{ }^{\circ}[\%]\end{array}$ & $\begin{array}{c}\text { A la } \\
\text { semana } \\
N .{ }^{\circ}(\%)\end{array}$ & $\begin{array}{c}\text { Al } \\
\text { mes } \\
N .{ }^{\circ}[\%]\end{array}$ & $D N 4^{b}$ & $\begin{array}{l}\text { Antes del } \\
\text { abordaje } \\
N .{ }^{\circ}[\%]\end{array}$ & $\begin{array}{c}\text { A la } \\
\text { semana } \\
N .{ }^{\circ}[\%]\end{array}$ & $\begin{array}{c}\text { Al } \\
\text { mes } \\
N .{ }^{\circ}[\%]\end{array}$ \\
\hline Leve & $12(8,4)$ & $111(77,6)$ & $101(77,6)$ & $<$ a 4 & $61(42,7)$ & $122(85,3)$ & $121(84,6)$ \\
\hline Moderado & $67(46,9)$ & $31(21,7)$ & $31(21,7)$ & $>$ a 4 & $82(57,3)$ & $21(14,7)$ & $21(14,7)$ \\
\hline Severo & $64(44,8)$ & $1(0,7)$ & $1(0.7)$ & & & & \\
\hline Total & 143 (100) & 143 (100) & $143(100)$ & & $143(100)$ & $143(100)$ & $143(100)$ \\
\hline
\end{tabular}

a: escala verbal análoga. b: cuestionario Douleur Neuropathique-4 items. 
Además, uno de los estudios en población mexicana sobre la persistencia de lumbalgia posterior a un procedimiento intervencionista es el de Cobarrubias y cols., quienes comunican una reducción significativa a través de la utilización de la escala numérica análoga [ENA] con un promedio de 5 a 6 posterior a la intervención en un año de seguimiento; sin embargo en el abordaje intervencionista solo se utilizó inyección peridural de esteroides. En este estudio se utilizaron y analizaron distintos abordajes intervencionistas, lo cual es de importancia ya que no existen estudios comunicados sobre pacientes oncológicos con lumbalgia (17).

De igual forma, la evaluación de DN4 en nuestro estudio mostró que más del 50 \% de los pacientes presentaban un puntaje mayor a 4 antes del abordaje y al mes posterior al mismo se observó un resultado menor a 4 puntos al mes de evaluación en el 84,6\% de la población. Castroman y cols. mencionan reducción del $50 \%$ del dolor neuropático en sus pacientes, utilizando técnicas y dosis similares a las de nuestro estudio, sin embargo las escalas de evaluación fueron distintas (18).

En cuanto a la respuesta obtenida a estos procedimientos se observó mejoría de más del $50 \%$ de dolor en el 86,7 \% de la población, datos similares a los encontrados en el estudio de Surbano y cols. (18); sin embargo, su seguimiento fue a corto plazo. En este estudio se realizó un control a la semana y al mes posterior al procedimiento, manteniendo resultados significativos que desencadenaron en la disminución del uso de opioides con una media de $22 \mathrm{mg} /$ día en el 35,7\% de los pacientes.

La lumbalgia es una patología dolorosa frecuente en la población en general y también en el paciente oncológico, y debido a la etiología múltiple de este tipo de padecimiento, la realización de diferentes abordajes en un mismo paciente pudo mejorar el dolor lumbar en gran proporción de pacientes, lo cual se vio reflejado en la disminución del consumo de analgésicos opioides. Sin embargo se requieren estudios prospectivos en el manejo intervencionista del dolor lumbar en población oncológica para mayor caracterización de su efectividad.

\section{AGRADECIMIENTOS}

Al personal de la clínica del dolor del Instituto Nacional de Cancerología por permitir realizar este estudio.

La presente investigación no ha recibido ayudas específicas provenientes de agencias del sector público, sector comercial o entidades sin ánimo de lucro.

\section{CONFLICTO DE INTERESES}

Los autores declaran que no tienen ningún conflicto de intereses.

\section{BIBLIOGRAFÍA}

1. Airaksinen O, Brox J, Cedraschi C, Hildebrandt J, Klaber J, Kovacs F, et al. Chapter 4. European guidelines for the management of chronic nonspecific low back pain. Eur Spine J. 2006;15(Suppl. 2):\$192-S300. DOI: 10.1007/s00586006-1072-1.

2. Hoy D, Brooks P, Blyth F, Buchbinder R. The Epidemiology of low back pain. Best Pract Res Clin Rheumatol. 2010;24(6):76981. DOl: 10.1016/j.berh.2010.10.002.

3. Waldman SD. Physical diagnosis of pain an atlas of signs and symptoms. 3. ${ }^{\text {a }}$ ed. Filadeldia: Elseivier; 2016. p. 221.

4. Batista J, Hernandez J, Nunez R, Pazos M, Aguirre J, Jreige $A$, et al. Prevalence of low back pain in Latin America: a systematic literature review. Pain Physician. 2014;17(5):379-91.

5. Chou R, Qaseem A, Snow V, Casey D, Cross T, Shekelle, et al. Diagnosis and Treatment of Low Back Pain: A Joint Clinical Practice Guideline from the American College of Physicians and the AmericanPain Society. Ann Intern Med. 2007;147(7):478-91. DOI: 10.7326/0003-4819-147-7200710020-00006.

6. Maher C, Underwood M, Buchbinder R. Non-specific low back pain. Lancet. 2016;389(10070):736-47. DOI: 10.1016/ S0140-6736(16)30970-9.

7. Deyo R, Diehl A. Cancer as a cause of back pain. J Gen Intern Med. 1988;3(3):230-8. DOI: 10.1007/BF02596337.

8. Wang D. Image Guidance Technologies for Interventional Pain Procedures: Ultrasound, Fluoroscopy, and CT. Curr Pain Headache Rep. 2018;22(1):6. DOI: 10.1007/s11916-0180660-1.

9. Steinberger J, Yuk F, Doshi A, Green S, Germano I. Multidisciplinary management of metastatic spine disease: initial symptom-directed management. Neurooncol Pract. 2020;7(Suppl. 1):33-44. DOl: 10.1093/nop/npaaO48.

10. Hatrick N, Lucas J, Timothy A, Smith M. The surgical treatment of metastatic disease of the spine. Radiother Oncol. 2000;56(3):335-9. DOI: 10.1016/S0167-8140(00)00199-7.

11. Perrin R, Laxton A. Metastatic spine disease: epidemiology, pathophysiology, and evaluation of patients. Neurosurg Clin N Am. 2004;15(4):365-73. DOI: 10.1016/j. nec.2004.04.018.

12. Ruiz S, Castellano M, Aparisi F. Papel de la radiología intervencionista en el diagnóstico y tratamiento de la columna vertebral dolorosa. Med Clin. 2013;140(10):458-65. DOI: 10.1016/j. medcli.2012.09.016.

13. Sayoa EB, Alvarez MA. Bases medico quirúrgicas en patología

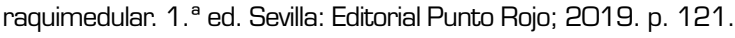

14. Kurita GP, Sjøgren P, Klepstad P, Mercadante S. Interventional Techniques to Management of Cancer-Related Pain: Clinical and Critical Aspects. Cancers. 2019;11(4):443. DOI: 10.3390/cancers11040443.

15. Mercadante S. Reviewing without a Clinical Background Is Detrimental for Cancer Pain Management. Cancers. 2019;11(7):1005. DOI: 10.3390/cancers11071005.

16. Surbano M, Antúnez M, Coutinho I, Machado V, Castromán P. Uso del Brief Pain Inventory (BPI) para la evaluación de las técnicas intervencionistas en el tratamiento de la lumbalgia. Dolor. 2014;23(62):10-4.

17. Covarrubias A. Lumbalgia: Un problema de salud pública. Rev Mex Anestesiol. 2010;33(Suppl. 1):106-9.

18. Castroman P, Surbano M, Alberti M, Shwartzmann A, Ayala S, Cristiani F. Inyección epidural de corticoides en el tratamiento del síndrome radicular lumbosacro (SRL). Anest Analg Reanim. 2015;28(2). 